\title{
Silencing of LncRNA-PVT1 ameliorates lipopolysaccharide-induced inflammation in THP-1-derived macrophages via inhibition of the p38 MAPK signaling pathway
}

\author{
Shengcai Zheng ${ }^{1,2,3 \#}$, Weichao $\mathrm{Li}^{1,2,3 \#}$, Wenhua Liao ${ }^{1,2,3}$, Canxia Huang ${ }^{1,2,3}$, Minggen Zhou ${ }^{1,2,3}$, \\ Yukai Zheng ${ }^{1,2,3}$, Zijun Zou ${ }^{1,2,3}$, Zhijie $\mathrm{He}^{1,2,3}$ \\ ${ }^{1}$ Guangdong Provincial Key Laboratory of Malignant Tumor Epigenetics and Gene Regulation, Sun Yat-sen Memorial Hospital, Sun Yat- \\ sen University, Guangzhou, China; ${ }^{2}$ RNA Biomedical Institute, Sun Yat-sen Memorial Hospital, Sun Yat-sen University, Guangzhou, China; \\ ${ }^{3}$ Department of Critical Care Medicine, Sun Yat-sen Memorial Hospital, Sun Yat-sen University, Guangzhou, China \\ Contributions: (I) Conception and design: S Zheng, W Li, Z He; (II) Administrative support: None; (III) Provision of study materials or patients: \\ None; (IV) Collection and assembly of data: S Zheng, Z Zou, C Huang; (V) Data analysis and interpretation: W Li, W Liao, M Zhou, Y Zheng; (VI) \\ Manuscript writing: All authors; (VII) Final approval of manuscript: All authors. \\ "These authors contributed equally to this work. \\ Correspondence to: Zhijie He. Department of Critical Care Medicine, Sun Yat-sen Memorial Hospital, Sun Yat-sen University, 107 Yan Jiang Road, \\ Guangzhou 510120, China. Email: hezhijie@mail.sysu.edu.cn; Zijun Zou. Department of Critical Care Medicine, Sun Yat-sen Memorial Hospital, \\ Sun Yat-sen University, 107 Yan Jiang Road, Guangzhou 510120, China. Email: zouzij@mail.sysu.edu.cn.
}

Background Sepsis is common in intensive care units and has a high mortality rate; yet, its pathogenesis and treatment remain unclear. Recent studies have shown that long non-coding RNA plasmacytoma variant translocation 1 (lncRNA-PVT1) plays a pro-inflammatory role in immune-related inflammatory diseases. Therefore, we investigated whether lncRNA-PVT1 plays an important pro-inflammatory effect in the inflammatory response of sepsis.

Methods: Quantitative real-time PCR (RT-qPCR) was employed for the detection of lncRNA-PVT1, interleukin $1 \beta$ (IL-1 $\beta$ ), and tumor necrosis factor $\alpha(T N F-\alpha)$ mRNA, and the correlations between their expressions were analyzed. After lncRNA-PVT1 knockdown by lncRNA Smart Silencer, abnormal expressions of lncRNA-PVT1, and IL- $1 \beta$ and TNF- $\alpha$ mRNA were detected. The expressions of total and phosphorylated protein of p38 were detected by western blotting. The effect of silencing lncRNA-PVT1 on p38 mitogen-activated protein kinase (MAPK) signaling pathway during lipopolysaccharide (LPS)induced inflammation was subsequently analyzed. The MAPK selective inhibitor, SB202190, was used to block this signaling pathway, and the expressions of lncRNA-PVT1 and TNF- $\alpha$ were detected by RT-qPCR. Furthermore, the effect of partial blockade of the p38 MAPK signaling pathway by SB202190 on the levels of lncRNA-PVT1 was explored.

Results: Following treatment of THP-1-derived macrophages with different concentrations of LPS, the levels of lncRNA-PVT1 and IL-1 $\beta$, TNF- $\alpha$ mRNA were increased in a dose-dependent manner. Silencing of lncRNA-PVT1 reduced the expressions of IL-1 $\beta$ and TNF- $\alpha$ mRNA via inhibition of the p38 MAPK signaling pathway. Specifically, inhibiting the p38 MAPK pathway significantly decreased the LPS-induced lncRNA-PVT1 elevation.

Conclusions: Our observations suggest that lncRNA-PVT1 can be silenced to ameliorate LPS-induced inflammation in macrophages via inhibition of the p38 MAPK pathway. Further, the p38 MAPK pathway can regulate the expression of lncRNA-PVT1 via a positive feedback loop.

Koywords: Sepsis; lncRNA; PVT1; macrophage

Submitted Mar 04, 2021. Accepted for publication Jun 04, 2021.

doi: 10.21037/apm-21-1078

View this article at: https://dx.doi.org/10.21037/apm-21-1078 


\section{Introduction}

Sepsis is a systemic inflammatory response syndrome which is triggered by severe infection. Patients with sepsis can suffer multiple organ dysfunction and may progress to septic shock (1). As a serious public health problem in both developed and developing countries with increasing populations of seniors and neonates, the World Health Organization (WHO) officially declared sepsis as a global health priority on May 26, 2017 (2). The pathological process of sepsis is complex and mainly involves an exaggerated inflammatory reaction. Currently, most sepsis research is primarily focused on whether better treatments can be achieved by using gene-level intervention and regulation during the development and progression of inflammation (3). During this process, numerous coding or non-coding genes display structural and expression abnormalities.

Long non-coding RNAs (lncRNAs), a group of noncoding RNA transcripts exceeding $200 \mathrm{nt}$ in length, regulate gene expression at the chromosomal, DNA, transcriptional, and post-transcriptional levels. In recent years, lncRNAs and their immune regulation in inflammatory diseases have become key research foci. There is increasing evidence indicating that lncRNAs regulate immune responses by regulating protein-protein interactions or DNA/RNA base pairing, thus affecting the differentiation and migration of immune cells and the secretion of inflammatory mediators $(4,5)$.

LncRNAs have become new regulatory targets, especially in the field of septic lung inflammation. Li et al. reported that the lncRNA maternally expressed gene 3 (MEG3) regulates transcript 4 (MEG3-4) during pulmonary bacterial infection, which allows for the binding of MEG3-4 to the microRNA miR-138, increased expression of interleukin (IL)-1 beta (1 $\beta)$ in lung macrophages and lung epithelial cells, and participation in pulmonary inflammation regulation (6). Huang et al. showed that the lncRNA nuclear-enriched abundant transcript 1 (NEAT1) has a higher expression level in patients with sepsis. Moreover, lncRNA NEAT1 was positively correlated with the Acute Physiology and Chronic Health Evaluation (APACHE) II score, serum tumor necrosis factor alpha (TNF- $\alpha$ ), IL$1 \beta$, IL 6, and IL-8, and was negatively correlated with IL10. This suggests that lncRNA NEAT1 increases the risk of sepsis and aggravates the severity of the disease, which is related to inflammatory factors (7). Huang et al. also found that lncRNA NEAT1 levels were significantly increased in the peripheral blood mononuclear cells (PBMCs) of their sepsis cohort compared to the healthy control group. LncRNA NEAT1 has exhibited high specificity in diagnosing sepsis and predicting disease prognosis, and may potentially serve as a serological marker (8).

Human chromosome $8 \mathrm{q} 24.21$ is home to the gene of lncRNA plasmacytoma variant translocation 1 (PVT1), which measures 1,716 bp in length. LncRNA-PVT1 is highly expressed in various tumor tissues or cell lines, as an important player in tumor development and tumorigenesis (9). The role of lncRNA-PVT1 in inflammation regulation has also been reported. Using a model of lipopolysaccharide (LPS)-induced acute kidney injury, Huang et al. showed that PVT1 regulated the c-Jun N-terminal kinase (JNK)/nuclear factor kappa $\mathrm{B}(\mathrm{NF}-\kappa \mathrm{B})$ pathway through its binding to TNF- $\alpha$, thereby promoting the inflammatory response (10). Hence, we hypothesize that PVT1 might be involved in the inflammatory response in septic acute lung injury (ALI).

The present work set out to investigate the differential lncRNA-PVT1 expressions in THP-1-derived macrophages treated with LPS. Knockdown of lncRNA-PVT1 can play a key anti-inflammatory role through inhibiting the p38 MAPK signaling pathway, which reversely blocks the expression of LPS-inducible LncRNA-PVT1. This research could provide a theoretical basis for further exploration of the mechanisms of lncRNA-PVT1 in inflammatory responses in sepsis. We present the following article in accordance with the MDAR reporting checklist (available at https://dx.doi.org/10.21037/apm-21-1078).

\section{Methods}

\section{Materials}

We purchased 12-myristate 13-acetate (PMA) and LPS from Escherichia coli O55:B5 from Sigma-Aldrich (St. Louis, Missouri, USA), and fetal bovine serum (FBS) and Roswell Park Memorial Institute 1640 (RPMI 1640) Medium from Gibco (Thermo Fisher Scientific, USA). SB202190, a p38 selective inhibitor, was obtained from Selleck (Houston, Texas, USA). The antibodies used in this study were obtained from Cell Signaling Technology (Beverly, MA, USA). We purchased an RNAiso Plus, PrimeScript $^{\mathrm{TM}}$ RT reagent Kit with gDNA Eraser and TB Green ${ }^{\mathrm{TM}}$ Premix Ex Taq II from Takara Bio (Dalian, China), and a bicinchoninic acid (BCA) protein assay kit and bovine serum albumin (BSA) from Beyotime Biotechnology (Shanghai, China). 


\section{Cell culture and treatment}

American Type Culture Collection (Manasas, VA, USA) supplied the THP-1 cells used in our experiments, which were first cultured using RPMI 1640 containing 10\% FBS and $1 \%$ penicillin-streptomycin, and incubated at $37{ }^{\circ} \mathrm{C}$ in $5 \% \mathrm{CO}_{2} / 95 \%$ air. The addition of PMA $(162 \mathrm{nM})$ was made to promote the differentiation of the monocytic THP-1 cells to macrophages $(11,12)$. The cells were left to differentiate for 48 hours after 12 hours of PMA treatment, and morphologic examination and total adhesion to the plate were used to verify their differentiation. Finally, LPS dissolved in growth medium was used for inflammation stimulation of the THP-1-derived macrophages.

\section{RNA extraction and quantitative real-time PCR}

RNAiso Plus was used to extract total RNA from the THP-1-derived macrophages. The PrimeScript ${ }^{\mathrm{TM}}$ RT reagent Kit with gDNA Eraser was used to generate highfidelity complementary DNA from all of the RNA samples individually. Quantitative real-time PCR (RT-qPCR) reaction samples were prepared as a mixture with $\mathrm{TB}$ Green ${ }^{\mathrm{TM}}$ Premix Ex Taq ${ }^{\mathrm{TM}}$ II (Takara Bio, Dalian, China) in line with the protocol supplied with the kit. Reactions were performed using a LightCycler ${ }^{\circledR} 480$ instrument (Roche, USA). GAPDH was used as an endogenous control. The $2^{-\Delta \Delta \mathrm{Ct}}$ method was applied for the relative quantification of lncRNA-PVT1, IL-1 $\beta$, TNF- $\alpha$, and GAPDH mRNA. Table S1 displays each primer sequence used for RT-qPCR.

\section{Western blot}

Total protein was extracted from the THP-1-derived cells. A BCA protein assay kit was used to determine protein concentrations. Sodium dodecyl sulphate-polyacrylamide gel $(12.5 \%)$ electrophoresis was carried out to separate $30 \mu \mathrm{g}$ protein from each of the different cell groups. Polyvinylidene difluoride membranes, on to which the separated proteins had been transferred, were subjected to blocking with $5 \%$ BSA for 1 hour at room temperature. This was followed by overnight incubation at $4{ }^{\circ} \mathrm{C}$. In the presence of the following primary antibodies: p-p38 $(1: 1,000), \mathrm{p} 38$ (1:1,000), and GAPDH $(1: 1,000)$. After washing, each membrane was once again subjected to incubation, this time with a secondary antibody $(1: 5,000)$ for 1 hour at room temperature. Following 3 washes with tris-buffered saline with Tween (TBST), the membranes were subjected to 1-hour incubation with horseradish peroxidase (HRP)-conjugated IgG (1:1,000). After washing the membranes for a final time, we used an imaging system (Bioworld, California, USA) to detect the protein bands. GAPDH was used to normalize the relative protein levels.

\section{LncRNA Smart Silencer transfection}

We accomplished knockdown of lncRNA-PVT1 expression by using LncRNA Smart Silencer, which was synthesized by RiboBio (Guangzhou, China) and comprises 3 siRNAs and 3 antisense oligonucleotides (ASOs). Table S2 shows the siRNA and ASO target sequences. Negative control (NC) Smart Silencer is free from domains homologous to human, mice, and rats. X-treme GENE siRNA Transfection Reagent (Roche, German) was employed to transfect cells with LncRNA Smart Silencer in line with the instructions supplied with the kit. LncRNA-PVT1 Smart Silencer was transfected at a concentration of $100 \mathrm{nM}$ with $\mathrm{X}$-treme GENE siRNA Transfection Reagent diluted in siRNA Transfection Medium. After 48 hours, we subjected the cells to stimulation with LPS or an identical amount of medium to support gene silencing. Cells were collected 8 hours after stimulation with or without LPS for RNA isolation and protein extraction.

\section{Statistical analysis}

Data were presented as mean \pm standard error of the mean based on at least 3 independent experiments. Oneway analysis of variance was applied to analyze all data. Correlations between study variables were calculated by Pearson correlation analysis. GraphPad Prism 7 (GraphPad Software Inc., La Jolla, CA, USA) was used for all statistical analysis. $\mathrm{P}<0.05$ was deemed to indicate statistical significance.

\section{Results}

\section{Expression of IncRNA-PVT1, and IL-1 $\beta$ and TNF- $\alpha$ mRNA in THP-1-derived macrophages during LPS- induced inflammatory response}

We analyzed the levels of lncRNA-PVT1, and IL-1 $\beta$ and TNF- $\alpha$ mRNA expressed by THP-1-derived macrophages treated with LPS at different concentrations and for different incubation times by performing RT-qPCR analysis. As shown in Figure $1 A$, at 8 hours after LPS 
A

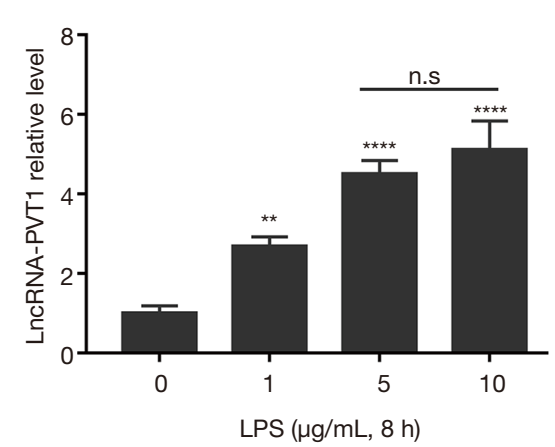

D

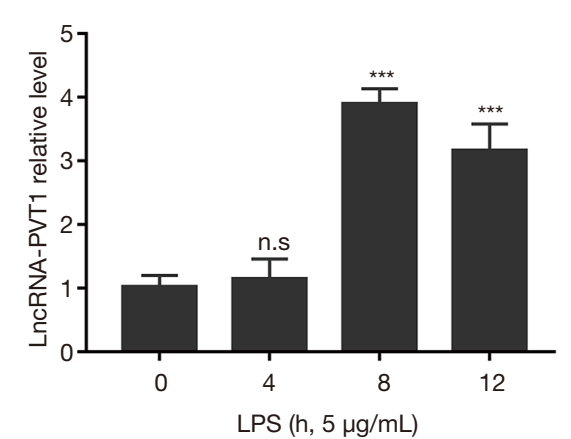

B

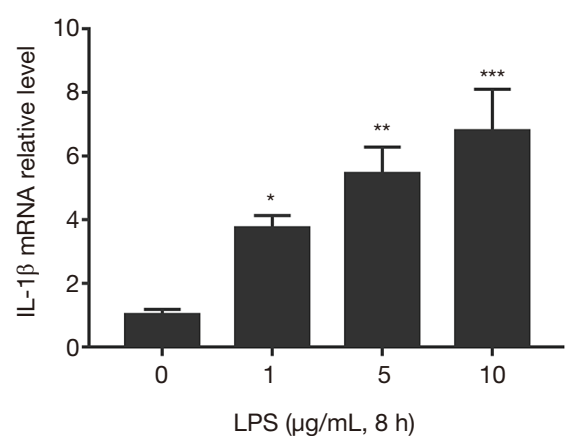

$\mathrm{E}$

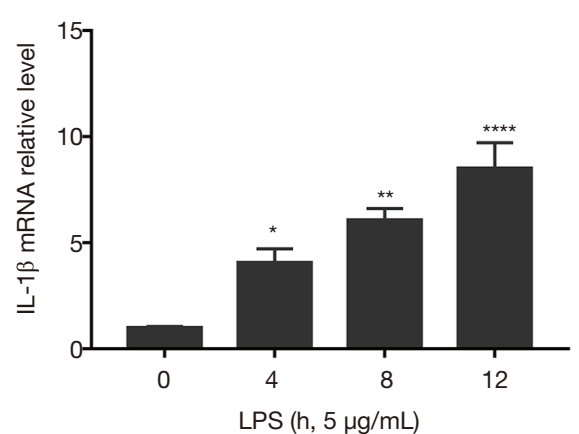

C

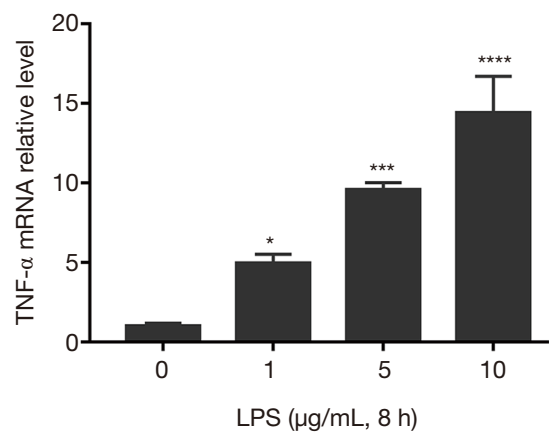

$\mathrm{F}$

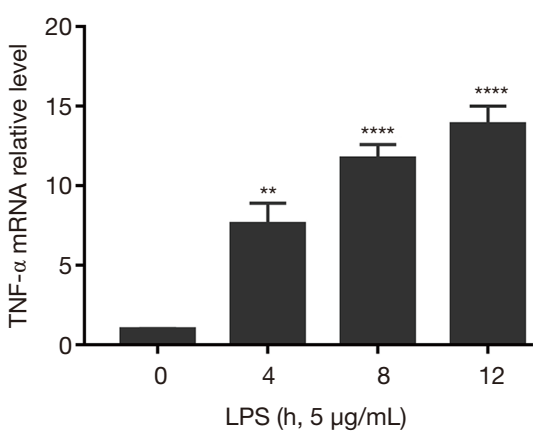

Figure 1 The expressions of lncRNA-PVT1 and IL-1 $\beta$, TNF- $\alpha$ mRNA in THP-1-derived macrophages during LPS-triggered inflammatory response. (A,B,C) THP-1-derived macrophages were subjected to treatment with LPS at various concentrations $(0,1,5$, and $10 \mu \mathrm{g} / \mathrm{mL}$ ) for 8 hours. (D,E,F) THP-1-derived macrophages were subjected to treatment with $5 \mu \mathrm{g} / \mathrm{mL}$ LPS for different lengths of time $(0,4,8$, and 12 hours). The expressions of lncRNA-PVT1, and IL-1 $\beta$ and TNF- $\alpha$ mRNA were analyzed through RT-qPCR. Quantitative data were presented as mean \pm SEM $(\mathrm{N}=3)$. One-way ANOVA was performed for comparison between multiple groups. In comparison to control, ${ }^{*} \mathrm{P}<0.05,{ }^{* *} \mathrm{P}<0.01,{ }^{* * *} \mathrm{P}<0.001,{ }^{* * * *} \mathrm{P}<0.0001$. IncRNA-PVT1, long non-coding RNA Plasmacytoma variant translocation 1; IL-1 $\beta$, interleukin $1 \beta$; TNF- $\alpha$, tumor necrosis factor $\alpha$; LPS, lipopolysaccharide; RT-qPCR, real-time quantitative polymerase chain reaction; SEM, standard error of the mean; n.s., no statistical difference.

stimulation, the level of lncRNA-PVT1 was increased in a dose-dependent manner. When LPS surpassed $5 \mu \mathrm{g} / \mathrm{mL}$, the increase of lncRNA-PVT1 expression exhibited no significant difference, regardless of the increase in LPS concentration. Similarly, the expressions of both IL-1 $\beta$ and TNF- $\alpha$ mRNA displayed an upward trend as the LPS concentrations increased (Figure 1B,C). When the LPS concentration was $5 \mu \mathrm{g} / \mathrm{mL}$, the expressions of IL- $1 \beta$ and TNF- $\alpha$ mRNA were also statistically significant compared with the control group $(\mathrm{P}<0.001)$. Thus, for subsequent functional experiments, we chose $5 \mu \mathrm{g} / \mathrm{mL}$ of LPS as the cell treatment concentration.

Cells treated with LPS for various incubation times exhibited a trend of rising and then declining expression of lncRNA-PVT1, which reached its highest level at 8 hours
(Figure 1D). Likewise, the expressions of IL-1 $\beta$ and TNF- $\alpha$ mRNA at 8 hours also exhibited a marked increase relative to the controls $(\mathrm{P}<0.01)$ (Figure $1 E, F)$. Therefore, in the following functional experiments, we chose 8 hours as the suitable incubation time for LPS-treated cells.

\section{Correlations of IncRNA-PVT1 with IL-1 $\beta$ and TNF- $\alpha$ mRNA expression in THP-1-derived macrophages during a LPS-induced inflammatory response}

To explore whether the expression of lncRNA-PVT1 is correlated with the expressions of IL- $1 \beta$ or TNF- $\alpha$ mRNA, a correlation analysis was performed to analyze their expressions in macrophages that had been differentiated from THP-1 cells and exposed to LPS at various concentrations 
A

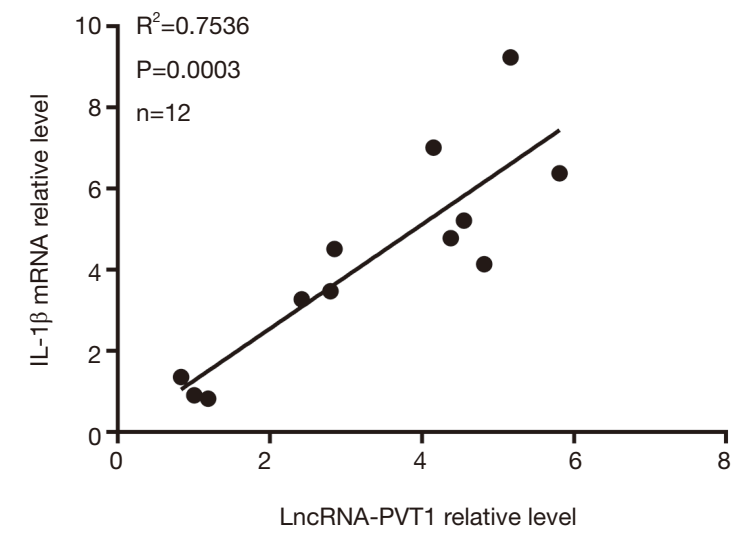

B

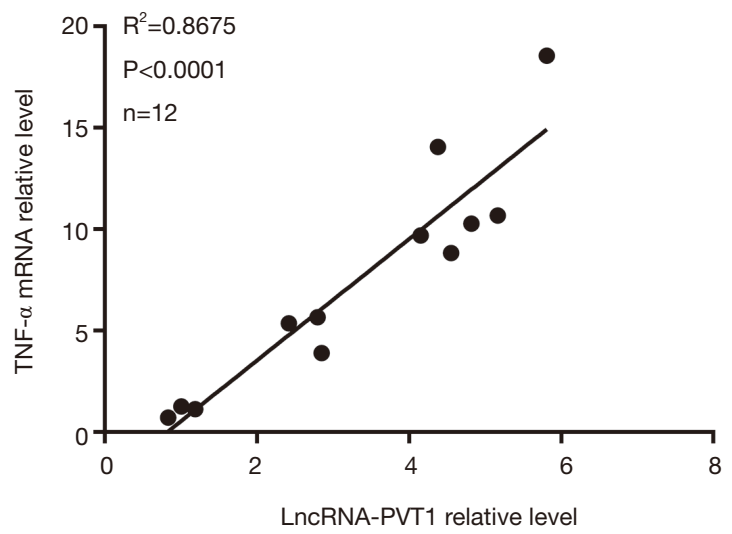

Figure 2 Co-expression of lncRNA-PVT1 and IL-1 $\beta$, TNF- $\alpha$ mRNA in THP-1-derived macrophages following stimulation with LPS at various concentrations $(0,1,5$, and $10 \mu \mathrm{g} / \mathrm{mL})$ for 8 hours. RT-qPCR was employed to detect the expressions of lncRNA-PVT1, and IL$1 \beta$ and TNF- $\alpha$ mRNA in LPS-induced macrophages. Correlation analysis between lncRNA-PVT1 expression and that of IL-1 $\beta$ mRNA (A) and TNF- $\alpha$ mRNA (B). Data are representative from three independent experiments. lncRNA-PVT1, long non-coding RNA Plasmacytoma variant translocation 1 ; IL-1 $\beta$, interleukin $1 \beta$; TNF- $\alpha$, tumor necrosis factor $\alpha$; LPS, lipopolysaccharide; RT-qPCR, real-time quantitative polymerase chain reaction.

for 8 hours. As Figure 2A,B shows, the levels of lncRNAPVT1 displayed positive correlations with the levels of IL- $1 \beta$ and TNF- $\alpha$ mRNA (both $\mathrm{P}<0.001$ ). Thus, we hypothesized that IncRNA-PVT1 might exert a positive regulatory effect on the expressions of both IL- $1 \beta$ and TNF- $\alpha$ mRNA by THP-1-derived macrophages under LPS induction.

\section{$I L-1 \beta$ and TNF- $\alpha$ mRNA levels after silencing of IncRNA- PVT1 in LPS-induced THP-1-derived macrophages}

To further confirm whether lncRNA-PVT1 has a positive regulatory effect on IL- $1 \beta$ and TNF- $\alpha$ mRNA expressions in LPS-induced inflammation, we subjected THP-1-derived macrophages to 48 hours of pre-treatment with $100 \mathrm{nM}$ LncRNA Smart Silencer followed by 8 hours of $5 \mu \mathrm{g} / \mathrm{mL}$ LPS stimulation. Then, RT-qPCR was performed to detect the expressions of lncRNA-PVT1, and IL- $1 \beta$ and TNF- $\alpha$ mRNA. We observed a significant decrease in lncRNAPVT1 expression after pre-treatment with LncRNA Smart Silencer, indicating that the silencer treatment was effective (Figure $3 A$ ). Furthermore, after lncRNA-PVT1 was knocked down effectively, the levels of IL- $1 \beta$ and TNF- $\alpha$ mRNA showed significant decreases after exposure to LPS compared to the control group (Figure 3B,C). These results suggested that the silencing of lncRNA-PVT1 could inhibit the expressions of IL- $1 \beta$ and TNF- $\alpha$ mRNA in LPSinduced THP-1-derived macrophages.

\section{The effects of lncRNA-PVT1 silencing on the p38 MAPK signaling pathway in LPS-induced THP-1-derived macrophages}

The p38 MAPK signaling pathway is known to be a critical player in LPS-stimulated inflammatory response. Yet, little is known about its role in LPS-induced inflammatory responses mediated by lncRNA-PVT1. To confirm the effect of lncRNA-PVT1 silencing on the p38 MAPK signaling pathway in LPS-challenged THP-1-derived macrophages, cells were pre-treated with LncRNA Smart Silencer followed by LPS stimulation. Western blotting was performed to detect the phosphorylation levels of the p38 protein, one of the critical signal proteins of MAPK pathway. As shown in Figure 4A,B, the Smart Silencer group exhibited a marked decrease in the phosphorylation levels of the $\mathrm{p} 38$ protein relative to the siRNA controls. Meanwhile, none of the groups showed a significant difference in the total levels of p38 (Figure 4C). These results suggested that silencing of lncRNA-PVT1 might inhibit the p38 MAPK signaling pathway, thereby inhibiting the upregulation of inflammatory genes in LPS-activated macrophages.

The expression of IncRNA-PVT1 after inbibiting the $p 38$ MAPK signaling patbway in LPS-induced THP-1-derived macrophages

The above results suggested that lncRNA-PVT1 expression 
A

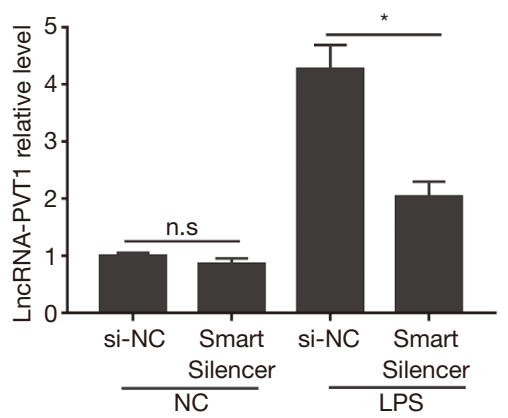

B

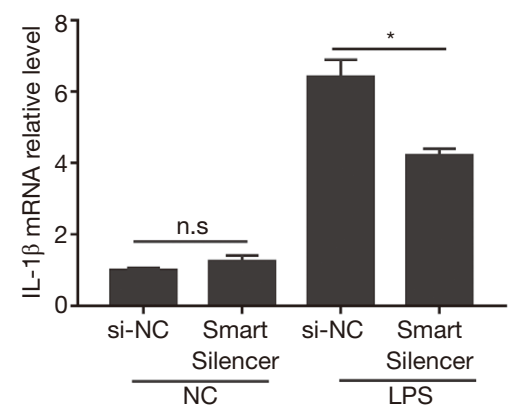

C

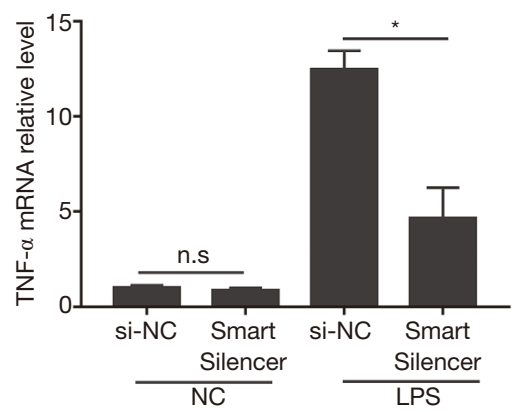

Figure 3 Silencing lncRNA-PVT1 can decrease the mRNA expressions of IL-1 $\beta$ and TNF- $\alpha$ in LPS-induced THP-1-derived macrophages. THP-1-derived macrophages were pre-treated with LncRNA Smart Silencer (100 nM, 48 hours) and then subjected to stimulation with LPS $(5 \mu \mathrm{g} / \mathrm{mL})$ for 8 hours. (A,B,C) The expressions of lncRNA-PVT1, and IL-1 $\beta$ and TNF- $\alpha$ mRNA were analyzed by RT-qPCR. Quantitative data were presented as mean $\pm \operatorname{SEM}(\mathrm{N}=3)$. Comparisons between 2 groups were conducted using 2-tailed Student's $t$ tests. Compared with control, ${ }^{*} \mathrm{P}<0.05$. NC, negative control; lncRNA-PVT1, long non-coding RNA Plasmacytoma variant translocation1; IL$1 \beta$, interleukin $1 \beta$; TNF- $\alpha$, tumor necrosis factor $\alpha$; LPS, lipopolysaccharide; si-NC, Smart Silencer NC; RT-qPCR, real-time quantitative polymerase chain reaction; SEM, standard error of mean.

A

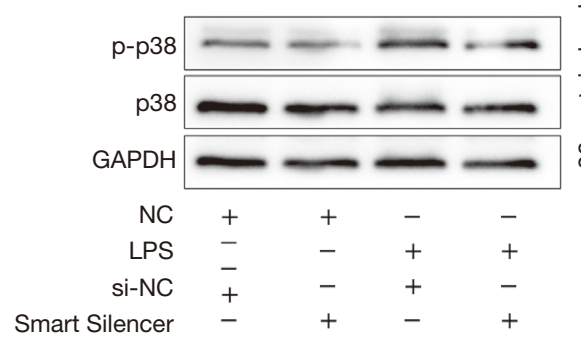

B

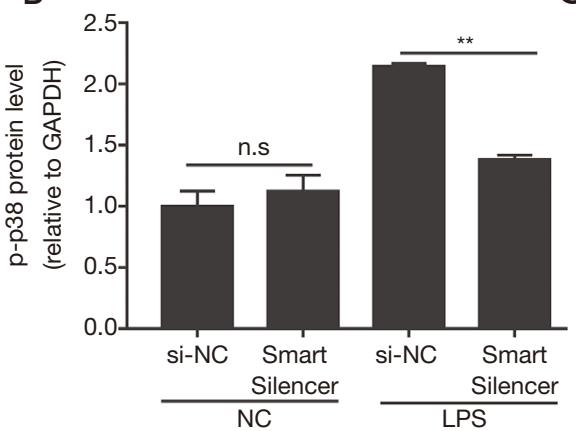

C

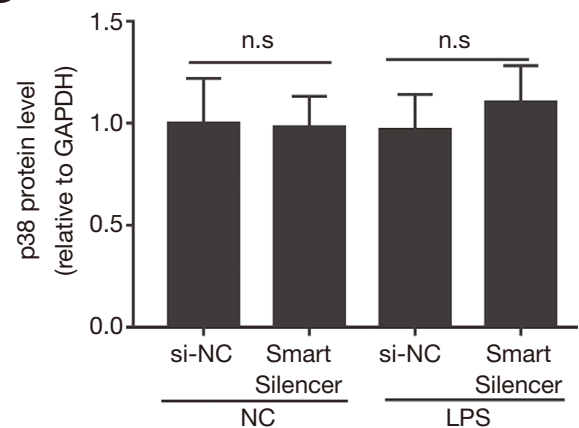

Figure 4 Silencing lncRNA-PVT1 might inhibit the p38 MAPK signaling pathway in LPS-induced THP-1-derived macrophages. THP1-derived macrophages were subjected to pre-treatment with LncRNA Smart Silencer (100 nM, $48 \mathrm{~h})$ and then stimulated with LPS in $5 \mu \mathrm{g} / \mathrm{mL}$ for 8 hours. (A,B,C) Western blotting was performed to analyze the phosphorylation levels and total levels of the p38 protein. Quantitative data were presented as mean \pm SEM $(\mathrm{N}=3)$. Comparisons between 2 groups were conducted using 2-tailed Student's $\mathrm{t}$ tests. Compared with control, ${ }^{* *} \mathrm{P}<0.01$. p-p38, phosphorylated p38; NC, negative control; LPS, lipopolysaccharide; si-NC, Smart Silencer NC; n.s., no statistical difference; lncRNA-PVT1, long non-coding RNA Plasmacytoma variant translocation1; MAPK, mitogen-activated protein kinase; RT-qPCR, real-time quantitative polymerase chain reaction; SEM, standard error of mean.

was upregulated, and silencing of lncRNA-PVT1 could ameliorate the LPS-induced inflammation via inhibition of the p38 MAPK signaling pathway. However, the mechanism of LPS-induced upregulation of lncRNAPVT1 was not clear. Therefore, we speculated whether the IncRNA-PVT1-regulated p38 MAPK pathway could exert partial effects on lncRNA-PVT1 expression via a feedback. To confirm our conjecture, we subjected macrophages differentiated from THP-1 cells to pre-treatment with the p38 selective pharmacological inhibitor SB202190 (10 $\mu \mathrm{M}, 2$ hours), and then to 8 hours of stimulation with LPS $5 \mu \mathrm{g} / \mathrm{mL}$. The TNF- $\alpha$ mRNA and lncRNA-PVT1 levels were then analyzed by RT-qPCR. As a downstream inflammatory signaling molecule of the p38 MAPK pathway, TNF- $\alpha$ mRNA expression was significantly decreased after pre-treatment with SB202190 followed by 

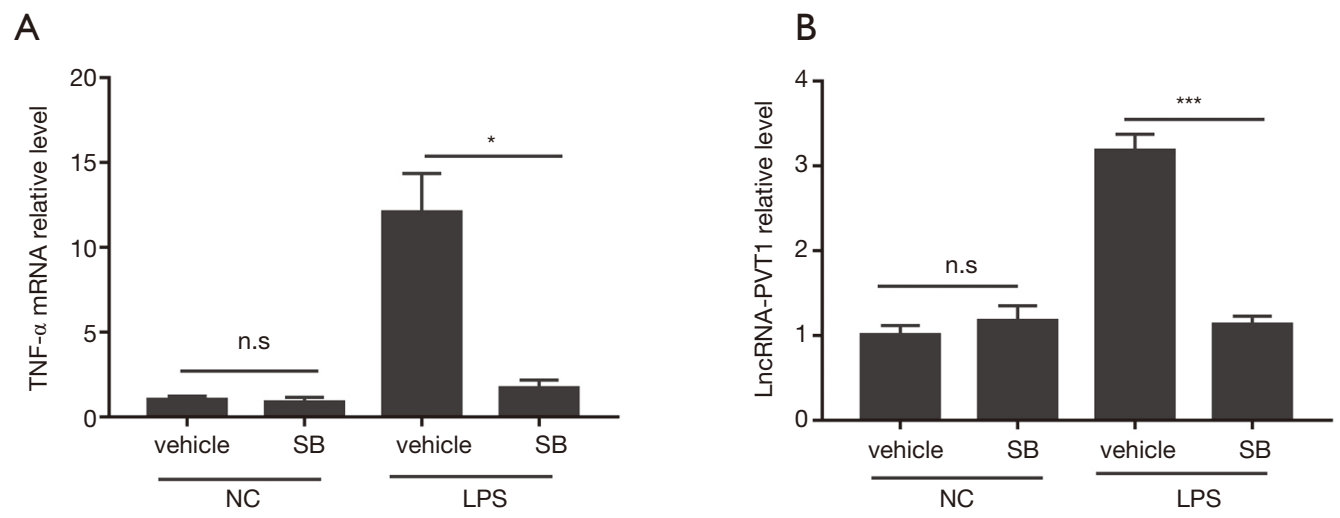

Figure 5 Effects of inhibiting the p38 MAPK pathway on TNF- $\alpha$ mRNA and lncRNA-PVT1 expression in LPS-induced THP-1-derived macrophages. THP-1-derived macrophages were subjected to 2 hours' pre-treatment with the p38 selective pharmacological inhibitor, SB202190 $(10 \mu \mathrm{M})$, followed by stimulation with LPS $(5 \mu \mathrm{g} / \mathrm{mL})$ for $8 \mathrm{~h}$. (A) The mRNA level of TNF- $\alpha$ was analyzed by RT-qPCR. (B) LncRNA-PVT1 expression was analyzed by RT-qPCR. Quantitative data were presented as mean $\pm \mathrm{SEM}(\mathrm{N}=3)$. Comparisons between 2 groups were conducted using 2 -tailed Student's t tests. In comparison to control, ${ }^{*} \mathrm{P}<0.05$, ${ }^{* * *} \mathrm{P}<0.001$. NC, negative control; LPS, lipopolysaccharide; SB, SB202190; n.s., no statistical difference; MAPK, mitogen-activated protein kinase; TNF- $\alpha$, tumor necrosis factor $\alpha$; lncRNA-PVT1, long non-coding RNA Plasmacytoma variant translocation1; RT-qPCR, real-time quantitative polymerase chain reaction; SEM, standard error of mean.

LPS stimulation (Figure $5 A$ ), indicating that the inhibition treatment was effective. Meanwhile, lncRNA-PVT1 expression was also notably decreased when the p38 MAPK pathway was effectively inhibited (Figure $5 B$ ). These results suggested that LPS-induced lncRNA-PVT1 expression in THP-1-derived macrophages is regulated by the p38 MAPK pathway in a positive feedback manner.

\section{Discussion}

Essentially, sepsis is an excessive inflammatory response mediated by various inflammatory cells (macrophages, neutrophils, and vascular endothelial cells), as well as their released inflammation-related factors, cytokines [such as TNF- $\alpha$, IL- $1 \beta$, IL-6, IL-8, platelet-activating factor (PAF), and inducible nitric oxide synthase (iNOS)] $(13,14)$. Inflammatory factors, including TNF- $\alpha$ and IL-1 $\beta$, are secreted by activated macrophages. Interactions between various inflammatory cytokines are key to the continuous progression of lung injury (15).

Most lncRNAs do not encode proteins. They are present in the nucleus or cytoplasm in the form of RNA and regulate protein-coding genes. LncRNAs regulate gene expression at multiple levels via a variety of mechanisms, and participate in pathological processes such as tumor formation, viral replication, and inflammatory injury $(16,17)$.
A growing bank of research has revealed a close relationship between IncRNAs and the development and prognosis of sepsis (18). Chen et al. discovered that metastasis-associated lung adenocarcinoma transcript 1 (MALAT1) is a regulator in the miR-125b and P38 mitogen-activated protein kinase (MAPK)/NF- $\kappa \mathrm{B}$ pathways in the aggravation of sepsisassociated chest infection. Consequently, MALAT1 became a potential target for the diagnosis and treatment of sepsis (19). Further, Jia et al.'s work documented LPS's effects of promoting lncRNA-CCL2 expression and increasing the release of inflammatory factors from macrophages by reducing the expression of sirtuin 1 (SIRT1) (20). Zhuang et al. found that IL-6 upregulated MALAT1 levels in LPSinduced septic cardiomyocytes. MALAT1 enhanced TNF- $\alpha$ expression by regulating serum amyloid antigen 3 (SAA3). MALAT1 upregulation is the mechanism responsible for LPS-induced cardiomyocyte death (21). Huang et al. found that lncRNA-PVT1 expression was significantly upregulated in a cell model of LPS-induced septic acute kidney injury. LncRNA-PVT1 promoted the release of IL- $1 \beta$ and other cytokines via the JNK/ NF- $\kappa B$ pathway, and its expression was significantly upregulated in acute kidney injury (10). However, the specific mechanisms of lncRNA-PVT1 in the LPS-induced inflammatory response remained unclear.

In this study, we established a model of the inflammatory 
response by stimulating THP-1 derived macrophages with LPS and compared the expressions of lncRNAPVT1 and IL-1 $\beta$, TNF- $\alpha$ mRNA among cells treated with various doses and duration of LPS. The levels of lncRNA-PVT1, and IL- $1 \beta$ and TNF- $\alpha$ mRNA were dosedependently increased; lncRNA PVT1 expression reached its highest level in cells treated with LPS for 8 hours, while IL- $1 \beta$ and TNF- $\alpha$ mRNA levels continued to rise time-dependently. The above findings indicated that the upregulation of lncRNA-PVT1 expression and elevation of the levels of inflammatory mediators (including IL$1 \beta$ and TNF- $\alpha$ mRNA) might jointly promote the LPSinduced inflammatory response in macrophages. Further investigation of the correlation between IncRNA-PVT1 and related inflammatory genes expression revealed that lncRNA-PVT1 expression displayed positive correlations with the expressions of IL- $1 \beta$ and TNF- $\alpha$ mRNA, which indicated that lncRNA-PVT1 participated in the LPSinduced inflammatory response in macrophages by upregulating the expression of the inflammatory factors IL$1 \beta$ and TNF- $\alpha$ mRNA.

It is well established that extracellular LPS of Gramnegative bacteria exerts a crucial role in aggravating an innate immune response and inflammation. Activation of the TLR4 signaling pathway by LPS subsequently engages p38 MAPK, ultimately leading to immune and inflammatory responses (22). Therefore, the mRNA levels of IL- $1 \beta$ and TNF- $\alpha$ in LPS-stimulated THP-1-derived macrophages were determined after silencing of lncRNAPVT1. The results showed that the expressions of the downstream inflammatory genes $I L-1 \beta$ and $T N F-\alpha$ were significantly downregulated after silencing of lncRNAPVT1. Meanwhile, the activity of the p38 MAPK pathway was decreased, suggesting that silencing of lncRNA-PVT1 might ameliorate LPS-induced inflammation in THP-1derived macrophages via inhibition of the p38 MAPK signaling pathway. To further confirm the mechanism underlying LPS-induced IncRNA-PVT1 up-regulation in this model, we detected the expression of IncRNA-PVT1 by specifically inhibiting the p38 MAPK pathway, and the results showed the lncRNA-PVT1 elevation in LPS-induced macrophages decreased markedly compared with the vehicle control.

By elucidating the role and mechanism of IncRNAPVT1 in LPS-induced cellular inflammatory response in macrophages, this study has provided a promising and novel target for gene-level intervention and regulation during the development and progression of inflammation, which is an important innovation in the field of septic lung injury research.
However, the mechanism by which the p38 MAPK signaling pathway regulates the upregulation of IncRNA-PVT1 expression was not clarified. Moreover, our data were based on in vitro experiments. Further experiments in clinical samples and animal models are necessary to verify our conclusion. Therefore, future studies should focus on the above 2 points in order to comprehensively elucidate the specific mechanisms of IncRNA-PVT1 in the inflammatory response of sepsis.

In conclusion, our results demonstrate that the silencing of lncRNA-PVT1, which was induced by LPS, might ameliorate the inflammation in THP-1-derived macrophages via inhibition of the p38 MAPK signaling pathway. We have also shown that the p38 MAPK pathway regulates lncRNA-PVT1 expression, possibly in a positive feedback manner.

\section{Acknowledgments}

Funding: This work was supported by the National Natural Science Foundation of China (81801945) and the Basic and Applied Basic Research of Guangdong (2020A1515010151).

\section{Footnote}

Reporting Checklist: The authors have completed the MDAR reporting checklist. Available at https://dx.doi. org/10.21037/apm-21-1078

Data Sharing Statement: Available at https://dx.doi. org/10.21037/apm-21-1078

Conflicts of Interest: All authors have completed the ICMJE uniform disclosure form (available at https://dx.doi. org/10.21037/apm-21-1078). The authors have no conflicts of interest to declare.

Ethical Statement: The authors are accountable for all aspects of the work in ensuring that questions related to the accuracy or integrity of any part of the work are appropriately investigated and resolved.

Open Access Statement: This is an Open Access article distributed in accordance with the Creative Commons Attribution-NonCommercial-NoDerivs 4.0 International License (CC BY-NC-ND 4.0), which permits the noncommercial replication and distribution of the article with the strict proviso that no changes or edits are made and the original work is properly cited (including links to both the 
formal publication through the relevant DOI and the license). See: https://creativecommons.org/licenses/by-nc-nd/4.0/.

\section{References}

1. Singer M, Deutschman CS, Seymour CW, et al. The Third International Consensus Definitions for Sepsis and Septic Shock (Sepsis-3). JAMA 2016;315:801-10.

2. Reinhart K, Daniels R, Kissoon N, et al. Recognizing Sepsis as a Global Health Priority - A WHO Resolution. N Engl J Med 2017;377:414-7.

3. Hotchkiss RS, Monneret G, Payen D. Sepsis-induced immunosuppression: from cellular dysfunctions to immunotherapy. Nat Rev Immunol 2013;13:862-74.

4. Heward JA, Lindsay MA. Long non-coding RNAs in the regulation of the immune response. Trends Immunol 2014;35:408-19.

5. Magagula L, Gagliardi M, Naidoo J, et al. Lnc-ing inflammation to disease. Biochem Soc Trans 2017;45:953-62.

6. Li R, Fang L, Pu Q, et al. MEG3-4 is a miRNA decoy that regulates IL-1beta abundance to initiate and then limit inflammation to prevent sepsis during lung infection. Sci Signal 2018;11:eaao2387.

7. Huang Q, Huang C, Luo Y, et al. Circulating lncRNA NEAT1 correlates with increased risk, elevated severity and unfavorable prognosis in sepsis patients. Am J Emerg Med 2018;36:1659-63.

8. Huang S, Qian K, Zhu Y, et al. Diagnostic Value of the lncRNA NEAT1 in Peripheral Blood Mononuclear Cells of Patients with Sepsis. Dis Markers 2017;2017:7962836.

9. Meyer KB, Maia AT, O'Reilly M, et al. A functional variant at a prostate cancer predisposition locus at $8 \mathrm{q} 24$ is associated with PVT1 expression. PLoS Genet 2011;7:e1002165.

10. Huang W, Lan X, Li X, et al. Long non-coding RNA PVT1 promote LPS-induced septic acute kidney injury by regulating TNFalpha and JNK/NF-kappaB pathways in HK-2 cells. Int Immunopharmacol 2017;47:134-40.

11. Stawowy P, Meyborg H, Stibenz D, et al. Furin-like proprotein convertases are central regulators of the membrane type matrix metalloproteinase-pro-matrix metalloproteinase-2 proteolytic cascade in atherosclerosis. Circulation 2005;111:2820-7.

12. Montoya-Rodríguez A, de Mejia EG, Dia VP, et al. Extrusion improved the anti-inflammatory effect of amaranth (Amaranthus hypochondriacus) hydrolysates in
LPS-induced human THP-1 macrophage-like and mouse RAW 264.7 macrophages by preventing activation of NFkappaB signaling. Mol Nutr Food Res 2014;58:1028-41.

13. Guo WA, Knight PR, Raghavendran K. The receptor for advanced glycation end products and acute lung injury/ acute respiratory distress syndrome. Intensive Care Med 2012;38:1588-98.

14. Goodman RB, Pugin J, Lee JS, et al. Cytokine-mediated inflammation in acute lung injury. Cytokine \& Growth Factor Reviews 2003;14:523-35.

15. Herold S, Mayer K, Lohmeyer J. Acute lung injury: how macrophages orchestrate resolution of inflammation and tissue repair. Front Immunol 2011;2:65.

16. Fatica A, Bozzoni I. Long non-coding RNAs: new players in cell differentiation and development. Nat Rev Genet 2014;15:7-21

17. Atianand MK, Caffrey DR, Fitzgerald KA. Immunobiology of Long Noncoding RNAs. Annu Rev Immunol 2017;35:177-98.

18. Ho J, Chan H, Wong SH, et al. The involvement of regulatory non-coding RNAs in sepsis: a systematic review. Crit Care 2016;20:383.

19. Chen H, Wang X, Yan X, et al. LncRNA MALAT1 regulates sepsis-induced cardiac inflammation and dysfunction via interaction with miR-125b and $\mathrm{p} 38$ MAPK/NFkappaB. Int Immunopharmacol 2018;55:69-76.

20. Jia Y, Li Z, Cai W, et al. SIRT1 regulates inflammation response of macrophages in sepsis mediated by long noncoding RNA. Biochim Biophys Acta Mol Basis Dis 2018;1864:784-92.

21. Zhuang YT, Xu DY, Wang GY, et al. IL-6 induced lncRNA MALAT1 enhances TNF- $\alpha$ expression in LPSinduced septic cardiomyocytes via activation of SAA3. Eur Rev Med Pharmacol Sci 2017;21:302-9.

22. Mandrekar P, Szabo G. Signalling pathways in alcoholinduced liver inflammation. J Hepatol 2009;50:1258-66.

(English Language Editor: A. Kassem)

Cite this article as: Zheng S, Li W, Liao W, Huang C, Zhou M, Zheng Y, Zou Z, He Z. Silencing of LncRNA-PVT1 ameliorates lipopolysaccharide-induced inflammation in THP-1-derived macrophages via inhibition of the $\mathrm{p} 38$ MAPK signaling pathway. Ann Palliat Med 2021;10(6):6410-6418. doi: 10.21037/apm-21-1078 
Supplementary

Table S1 Sequence of primers used in current investigation in RT-qPCR

\begin{tabular}{lcc}
\hline Gene & Forward (5'-3') & Reverse (5'-3') \\
\hline PVT1 & TGAGAACTGTCCTTACGTGACC & AGAGCACCAAGACTGGCTCT \\
$I L-1 \beta$ & ATGATGGCTTATTACAGTGGCAA & GTCGGAGATTCGTAGCTGGA \\
$T N F-\alpha$ & CCTCTCTCTAATCAGCCCTCTG & GAGGACCTGGGAGTAGATGAG \\
GAPDH & GCACCGTCAAGGCTGAGAAC & TGGTGAAGACGCCAGTGGA \\
\hline
\end{tabular}

Table S2 Ribo ${ }^{\text {TM }}$ LncRNA Smart Silencer for human lncRNA-PVT1 containing three siRNAs and three antisense oligonucleotides

\begin{tabular}{ll}
\hline Name & Sequence \\
\hline siRNA-1 target sequence & CCCAACAGGAGGACAGCTT \\
siRNA-2 target sequence & GCTGAATGCCTCATGGATT \\
siRNA-3 target sequence & CACTGAGGCTACTGCATCT \\
antisense oligonucleotides target sequence-1 & CAAGCACCTGTTACCTGTCC \\
antisense oligonucleotides target sequence-2 & TGGGCTTGAGCTGACCATAC \\
antisense oligonucleotides target sequence-3 & AGGAGCTGCATCTACCCTGC \\
\hline
\end{tabular}

